\title{
Prognostic Value of the Diagnostic Criteria Distinguishing Endometrial Stromal Sarcoma, Low Grade From Undifferentiated Endometrial Sarcoma, 2 Entities Within the Invasive Endometrial Stromal Neoplasia Family
}

\author{
Weiwei Feng, M.D., Ph.D., Anais Malpica, M.D., Stanley J. Robboy, M.D., Einar Gudlaugsson, M.D., \\ Keqin Hua, M.D., Ph.D., Xianrong Zhou, M.D., and \\ Jan P. A. Baak, M.D., Ph.D., F.R.C.Path., F.I.A.C(Hon)., F.I.C.P, DrHC (Antwerp).
}

\begin{abstract}
Summary: The World Health Organization (WHO 2003) recognizes 3 endometrial stromal neoplasms: noninvasive endometrial stromal nodule and the 2 invasive neoplasms, endometrial stromal sarcoma (ESS), low grade and undifferentiated endometrial sarcoma (UES). It is important to note that the WHO 2003 does not define moderate atypia (an important differentiating diagnostic criterion for ESS, low grade and UES), nor does it discuss its significance. Moreover, studies on reproducibility and additional prognostic value of other diagnostic features in large are lacking. Using strict definitions, we analyzed the agreement between routine and expert-review necrosis and nuclear atypia in 91 invasive endometrial stromal neoplasias (IESN). The overall 5-year and 10-year recurrence-free survival rate estimates of the 91 IESN patients were $82 \%$ and $75 \%$, respectively. Necrosis was well reproducible, and nuclear atypia was reasonably well reproducible. The 10-year recurrence-free survival rates for necrosis absent/inconspicuous versus prominent were $89 \%$ and $45 \%$ $(P<0.001)$ and those for review-confirmed none/mild, moderate, severe atypia were $90 \%, 30 \%$, and $<20 \%(P<0.00001)$. Therefore, cases with moderate/severe atypia should be grouped together. Nuclear atypia and necrosis had independent prognostic values (Cox regression). Once these features were taken into account, no other feature had an independent additional prognostic value, including mitotic count. Using "none/
\end{abstract}

From the Department of Gynecology and Shanghai Key Laboratory of Female Reproductive Endocrine-Related Diseases (W.F., K.H.); Department of Pathology (X.Z.), Obstetrics and Gynecology Hospital of Fudan University, Shanghai, China; Department of Pathology (E.G., J.P.A.B.), Stavanger University Hospital, Stavanger, Norway; Departments of Pathology and Gynecologic Oncology (A.M.), The University of Texas M.D. Anderson Cancer Center, Houston, TX; and Departments of Pathology and Obstetrics and Gynecology (S.J.R.), Duke University Medical Center, Durham, NC.

Supported by "211" key discipline construction project, China and in part by a grant to Jan P. A. Baak from the Stichting Bevordering Diagnostische Morfometrie, the Netherlands. The co-authors from Norway regret that due to a misinterpretation of the Norwegian health research law effective per July 1, 2009, they assumed that the approval of the study by the IRB of the Fudan University Obstetrics and Gynecology hospital was sufficient for them to participate in this retrospective non-interventional prognostic study on Chinese patients. As a result, they erroneously did not ask formal permission from the Norwegian Regional Ethics Committee (REC) and the Research Director of the Stavanger University Hospital to approve and get permission to participate in this study. However, in August and September 2011 the REC and the Research Director of the SUH decided after extensive considerations not to object against participation in the study and allowed the study to continue. Moreover, in September 2012 the Norwegian Health Inspection decided not to persecute the Norwegian authors and close the case.

Address correspondence and reprint requests to Dr Weiwei Feng, MD, PhD, Department of Gynecology, Obstetrics and Gynecology Hospital of Fudan University, 419 Fang Xie Road, Shanghai, China. E-mail: jingsakura@gmail.com. 
mild atypia, necrosis absent/inconspicuous" as ESS, low grade versus "moderate/severe atypia present or necrosis present" as UES resulted in $68 \mathrm{ESS}$, low grade and 23 UES cases with disease-specific overall mortality-free survival of $99 \%$ versus $48 \%$ $(P<0.00001$, hazard ratio $=45.4)$. When strictly defined microscopic criteria are used, the WHO 2003 diagnoses of ESS, low grade and UES are well reproducible and prognostically strong. Key Words: Atypia-Endometrial stromal sarcoma, low gradeNecrosis - Reproducibility_Prognosis-Undifferentiated endometrial sarcoma.

Invasive endometrial stromal neoplasms account for $15 \%$ of all uterine sarcomas and for approximately $0.2 \%$ to $0.7 \%$ of all uterine malignancies $(1,2)$. Originally, endometrial stromal sarcoma (ESS) was low grade or high grade based on mitotic count (3). Subsequently, in 1982, Evans (4) proposed a change in the paradigm used to approach this disease, which was later endorsed by other investigators and ultimately accepted by the World Health Organization classification (WHO 2003). ESS, low grade refers to densely cellular, invasive endometrial stromal tumors that lack both significant cellular atypia and pleomorphism. The stroma usually displays a rich network of small capillaries and arterioles. Necrosis is typically absent or inconspicuous, but vessel invasion can often be observed (1). ESS, low grade should be distinguished from endometrial stromal nodule, which lacks infiltrative margins. It should be distinguished also from undifferentiated endometrial sarcoma (UES), which is a high-grade sarcoma that lacks specific differentiation (without histologic resemblance to endometrial stroma), shows significant cellular atypia, and often but not always has prominent necrosis and hemorrhage (1). The behavior of ESS, low grade is indolent, although late recurrence and distal metastasis may occur. In contrast, UES has a much worse prognosis. Cases exist showing transition of ESS into high-grade sarcomas (5).

As the definitions of ESS before and after 2003 differ, studies on prognostic factors before 2003 cannot be applied to ESS, low grade diagnosed later. The problem with regard to earlier studies is that the various conditions were aggregated, preventing meaningful analysis of any single variable (6-13). Further, some recent studies are still using the older classification (14-27). Another problem is that the WHO 2003 criteria for the essential features to distinguish ESS, low grade and UES (necrosis and cellular atypia) are rather vaguely defined. Finally, the WHO 2003 does not at all describe the significance of moderate atypia. As a result, knowledge about the reproducibility and validity of diagnostic and prognostic criteria for the WHO 2003 classification is rather limited and uncertain, as the WHO 2003 also acknowledges that "A small minority of cases share features of low-grade ESS and undifferentiated sarcoma, and their classification is controversial."

We therefore set out to study in a large number of WHO 2003-defined cases with long follow-up the reproducibility and prognostic value of the essential diagnostic features. Using strict diagnostic criteria, necrosis (absent/inconspicuous vs. prominently present) and atypia (no or mild/moderate/severe) were compared. Special attention was paid to cases with moderate atypia. In addition, using multivariate survival analysis, this study tests whether features often mentioned as discriminatory (vascular invasion, tumor diameter, age, FIGO 2009 stage) have independent additional prognostic value. Finally, we studied the core of the WHO 2003 classification itself, whether ESS, low grade and UES are distinct and separate conditions with regard to their clinical behavior.

\section{PATIENTS AND METHODS}

The study was approved by the Institutional Review Board of the Fudan University Obstetrics and Gynecology Hospital. The patient cohorts have been described in detail before (28). In brief, the pathology files at that hospital contained 91 cases of invasive low-grade and high-grade endometrial stromal neoplasms according to the pre-WHO 2003 criteria, the diagnoses of which were independently confirmed by 2 experienced gynecologic pathologists (Xianrong Zhou, Jan P. A. Baak). Stage was determined using the 2009 FIGO rules for staging of endometrial and leiomyosarcomas (29,30). Diagnostic criteria per WHO 2003 assessed the absence or presence of prominent necrosis and the degree of nuclear atypia, which we assessed using the features proposed by Kempson and Hendrickson (31). Tumor cell necrosis, as opposed to other forms of necrosis, shows a relatively abrupt transition between necrotic and preserved cells. The nuclear ghost outlines of the 
necrotic cells can often be seen throughout the necrotic area, and inflammatory cells are uncommon. Necrosis is usually conspicuous in UES, the size exceeding more than half of a field of vision with a 40 times objective (about 0.4 to $0.6 \mathrm{~mm}$ in diameter). Assigning the degree of atypia took into account the degree of nuclear pleomorphism, nuclear size, nuclear membrane irregularities, chromatin density, and nucleolar size and prominence. Mildly (insignificantly) atypical cells show minimal variation in nuclear size and shape, and nucleoli are small. Moderate atypia shows scattered pleomorphic nuclei. Severe atypia shows many and severely pleomorphic nuclei. As only few cases showed no nuclear atypia, we grouped the atypia as none/mild. For analysis, we thus distinguished none/mild and separately distinguished moderate and severe atypia. Although moderate and severe atypia were evaluated separately, whenever appropriate they have been reported together as moderate/severe. Figure 1 gives typical microscopic examples of WHO2003 ESS, Low Grade and UES. Vessel invasion required the presence of tumor cells surrounded by a clear space lined with endothelial cells. Shrinkage artifact and true vessel invasion required a discrepancy of the shape of the space and tumor cells. In rare cases of doubt, CD10 immunohistochemistry was used.

Although the WHO 2003 no longer considers mitotic activity as a diagnostic criterion for the distinction of ESS, low grade and UES, we still assessed the number of mitoses in 10 fields of vision with a $\times 40$ objective, field diameter of $450 \mu \mathrm{m}$, and numeric aperture of 0.65 , using the Multicenter Morphometric Mammary Carcinoma Project protocol for counting mitoses (32).

\section{Statistic Analysis}

SPSS version 15 (SPSS; Chicago, IL) was used for the statistical analyses. To study the reproducibility of necrosis and cellular atypia, cross tables were constructed for these features, as mentioned in the pathology reports routinely made and as assessed by the reviewing gynecologic pathologists. Cross tables were also constructed for stage on the one hand and for necrosis and cytologic atypia on the other. The percentage and significance of overall agreement were calculated. The prognostic significance of the variables was assessed using the Kaplan-Meier survival analysis, with recurrence-free survival and overall disease-related survival as end points. The hazard ratios (HRs) and 95\% confidence intervals of the
TABLE 1. Recurrence-free prognostic value of FIGO stage of all malignant endometrial stromal neoplasms (inclusive of ESS, low grade and UES)

\begin{tabular}{lcccc}
\hline & & \multicolumn{3}{c}{ Percentage alive and well } \\
\cline { 3 - 5 } FIGO 2009 & $\begin{array}{c}\text { Total } \\
\text { number }\end{array}$ & $\begin{array}{c}\text { No. } \\
\text { stage }\end{array}$ & $\begin{array}{c}\text { At 5r } \\
\text { follow-up } \\
(\%)\end{array}$ & $\begin{array}{c}\text { At 10 yr } \\
\text { follow-up (\%) }\end{array}$ \\
\hline 1 & 69 & 10 & 86 & 82 \\
2 & 16 & 4 & 75 & 75 \\
3 & 6 & 4 & 33 & 33 \\
4 & 0 & 0 & - & -75 \\
Overall & 91 & 18 & 82 & 75 \\
\hline
\end{tabular}

various features were calculated by univariate regression analyses (Cox, Forward, and Wald). Multivariate regression (the Cox model) was used to assess the independent additional prognostic value of the different features studied.

\section{RESULTS}

The patients were 19 to 66 years of age (median, $43 \mathrm{yr}$ ). With a median follow-up of 5.8 years (range, 0.5 to $39 \mathrm{yr}$ ), there were 18 recurrences and 13 tumorrelated deaths. The Kaplan-Meier estimates of the 5 -year and 10-year recurrence-free survival rates were $82 \%$ and $75 \%$, respectively. For the 91 cases, the original researchers and reviewers agreed completely about necrosis (absent or inconspicuous vs. prominently present), which is an important criterion for the diagnosis of ESS, low grade versus UES. Nuclear atypia was only partially reproducible $(86 \%$ agreement), especially moderate atypia (29\% agreement between the original routine and the review diagnoses). However, the reviewing pathologists (using the strict criteria for moderate atypia mentioned above) agreed on moderate atypia in all cases (after discussion and careful comparison with other normal endometrial stroma and with mildly, moderately, or severely atypical cases, using a double-headed microscope).

Prognosis, as might be expected, is related to stage. Most of the tumors were at Stage $1(86 \%)$, and the prognosis was good $(88 \%$ at $5 \mathrm{yr}$ and $82 \%$ at $10 \mathrm{yr})$,

TABLE 2. Relationship between FIGO stage and necrosis $(P=0.004)$ for all malignant endometrial stromal tumors

\begin{tabular}{llr}
\hline & \multicolumn{2}{c}{ Necrosis } \\
\cline { 2 - 3 } FIGO & Present & Absent \\
\hline 1 & $6(9 \%)$ & $63(91 \%)$ \\
2 & $2(12 \%)$ & $14(88 \%)$ \\
3 & $1(17 \%)$ & $5(83 \%)$ \\
4 & 0 & 0 \\
\hline
\end{tabular}


TABLE 3. Relationship between FIGO stage and atypia $(P=0.002)$ for all malignant endometrial stromal tumors

\begin{tabular}{lcll}
\hline & \multicolumn{3}{c}{ Atypia } \\
\cline { 2 - 4 } FIGO 2009 stage & None/mild & Moderate & Severe \\
\hline 1 & $61(89 \%)$ & $5(7 \%)$ & $3(4 \%)$ \\
2 & $14(88 \%)$ & $0(0 \%)$ & $2(12 \%)$ \\
3 & $3(50 \%)$ & $2(33 \%)$ & $1(17 \%)$ \\
\hline
\end{tabular}

whereas the Stage 2 and 3 cases more often showed recurrence $(P<0.0001)$ (Stage $2,75 \%$ and $75 \%$; Stage $3: 33 \%$ and $33 \%$, at $5 \mathrm{yr}$ and $10 \mathrm{yr}$, respectively) (Table 1). Tables 2 and 3 show correlations between stage of necrosis and atypia. Necrosis increased from $9 \%$ in Stage 1 to $17 \%$ in Stage $3(P=0.004)$. Moderate and severe atypia also occurred significantly more often in Stages 2 and $3(P=0.04)$. Nuclear atypia and necrosis were significantly correlated $(P=0.002)$, with moderate and severely atypical tumors showing necrosis more often (Table 4), even though no prediction could be made in an individual case. Analysis of the mitotic count showed that 66 of the 68 WHO 2003-defined ESS, low grade had values $<10 / 10$ high-power fields. In contrast, the majority of UES $(56 \%)$ had values $\geq 10 / 10$ highpower fields.

Multivariate survival analysis (Cox model) of all features showed that atypia and necrosis had independent prognostic values (Table 5). The combination of atypia and necrosis is significantly stronger than any individual feature alone. The 5-year and 10 -year recurrence-free survival rates for necrosis absent/inconspicuous were $89 \%$ and $80 \%$, respectively, and those for prominent necrosis were $45 \%$ and $45 \%$, respectively $P<0.001$, (Fig. 2). For mild atypia, the 5-year and 10-year recurrence-free survival rates were $92 \%$ and $86 \%$, and for moderate atypia $29 \%$ and $29 \%$, respectively. For cases with
TABLE 4. Correlation between atypia and necrosis in the 91 cases $(P=0.002)$ for all malignant endometrial stromal tumors

\begin{tabular}{lrr}
\hline & \multicolumn{2}{c}{ Necrosis } \\
\cline { 2 - 3 } & Absent or inconspicuous & Prominent \\
\hline Atypia & $68(87 \%)$ & $10(13 \%)$ \\
Mild & $5(71 \%)$ & $2(29 \%)$ \\
Moderate & $2(33 \%)$ & $4(67 \%)$ \\
Severe & & \\
\hline
\end{tabular}

severe atypia, the last patient was followed up for 5.3 years, and therefore 10-year follow-up was not reached. The 5-year recurrence-free survival rate was $17 \% P<0.00001$, (Fig. 3). Therefore, the most impressive prognostic difference is between cases with none/mild versus moderate/severe atypia. In fact, the current survival data indicated that cases with moderate and severe atypia were prognostically not different from each other and should be grouped together against cases with none or mild atypia (Table 6), a conclusion drawn from univariate and multivariate proportional HR analyses (the Cox model).

Combinations of nuclear atypia and prominence of necrosis were evaluated for the most significantly prognostic groupings (Table 7). The first classification rule was "no + mild + moderate atypia combined with absent or inconspicuous necrosis" as ESS, low grade, versus all other cases with "either prominent necrosis present, or prominent necrosis absent but with severe atypia" as UES. This rule resulted in 73 ESS, low grade and 18 UES cases, with 10-year recurrence-free survival rates of $89 \%$ and $44 \%$, respectively $(P<0.0001, \mathrm{HR}=7.1)$ and overall mortality-free survival of the ESS, low grade and UES according to this rule of $95 \%$ and $50 \%$, respectively $(P<0.0001, \mathrm{HR}=11.6)$. The second rule differed

TABLE 5. Multivariate survival analysis (the Cox model) for all malignant stromal tumors

\begin{tabular}{|c|c|c|c|c|c|c|c|}
\hline \multirow[b]{2}{*}{ Step } & \multirow[b]{2}{*}{ Features } & \multirow[b]{2}{*}{ Beta } & \multirow[b]{2}{*}{ Standard error } & \multirow[b]{2}{*}{ Significance } & \multirow[b]{2}{*}{ Hazard ratio } & \multicolumn{2}{|c|}{$95 \%$ Confidence interval for hazard ratio } \\
\hline & & & & & & Lower & Upper \\
\hline \multicolumn{8}{|c|}{ Features in the equation } \\
\hline 1 & Atypia & 2.6 & 0.49 & $<0.0001$ & 13.0 & 5.0 & 33.5 \\
\hline \multirow[t]{2}{*}{2} & Necrosis & 1.5 & 0.50 & 0.003 & 4.3 & 1.6 & 11.3 \\
\hline & Atypia & 2.3 & 0.51 & $<0.0001$ & 9.9 & 3.7 & 27.0 \\
\hline \multicolumn{8}{|c|}{ Features not in the equation } \\
\hline Step & Feature & Significance & & & & & \\
\hline \multirow[t]{3}{*}{1} & Necrosis & 0.002 & & & & & \\
\hline & FIGO 2009 stage & 0.22 & & & & & \\
\hline & Vascular invasion & 0.02 & & & & & \\
\hline \multirow[t]{2}{*}{2} & FIGO 2009 stage & 0.15 & & & & & \\
\hline & Vascular invasion & 0.83 & & & & & \\
\hline
\end{tabular}



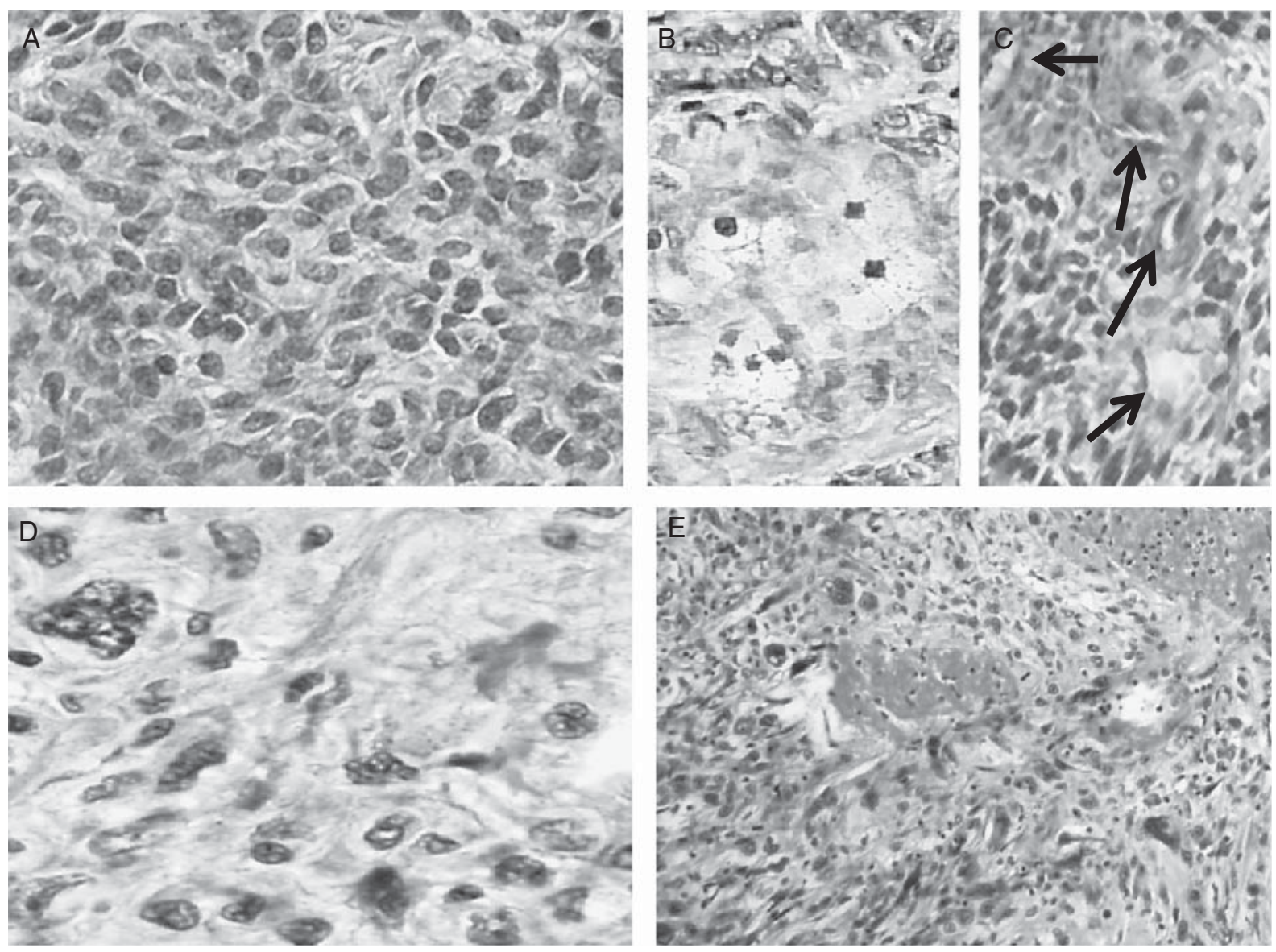

FIG. 1. Invasive endometrial stromal tumors. Top: Endometrial stromal sarcoma, low grade $(\mathrm{ESS})$ : $(\mathrm{A})=\mathrm{Cytology}$, $(\mathrm{B})=$ Foam cells, $(C)=$ delicate vascular structure (arrows). Bottom: Undifferentiated endometrial sarcoma (UES): (D) = Severe atypia, $(E)=$ tumor necrosis.

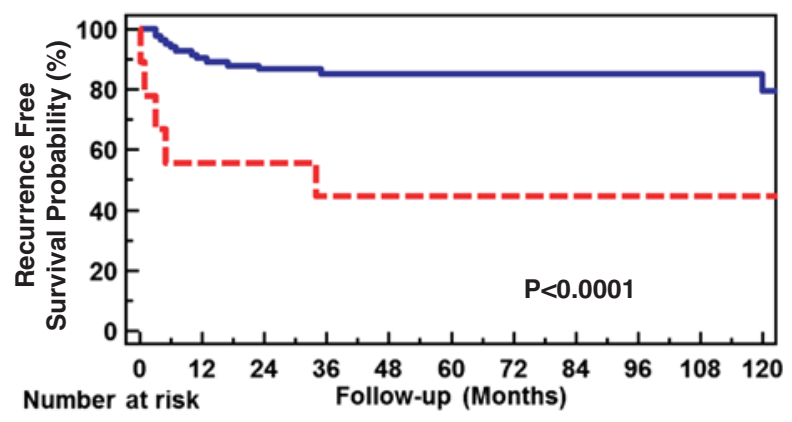

Necrosis Absent/Inconspicuous

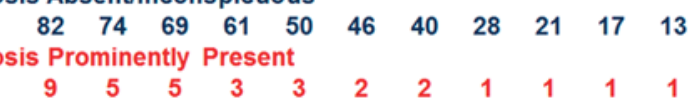

FIG. 2. The Kaplan-Meier 10-year recurrence-free (disease specific) survival curves of cases with necrosis absent/inconspicuous $(80 \%)$ versus prominently present $(45 \%)$ in the 91 endometrial stromal tumors, low grade.

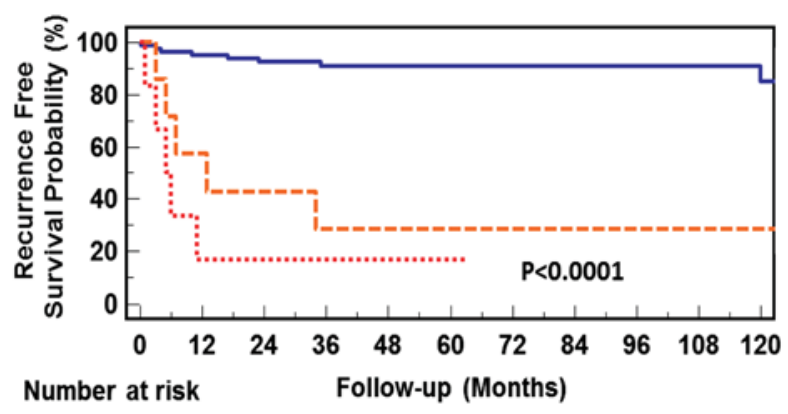

None/Mild Atypia

$\begin{array}{ccccccccccc}7874 & 70 & 61 & 50 & 46 & 41 & 28 & 21 & 17 & 13 \\ \text { Moderate Atypia } & & & & & & & & & \\ 7 & 4 & 3 & 2 & 2 & 1 & 1 & 1 & 1 & 1 & 1 \\ \begin{array}{c}\text { Severe Atypia } \\ 6\end{array} 1 & 1 & 1 & 1 & 1 & 0 & 0 & 0 & 0 & 0\end{array}$

FIG. 3. The Kaplan-Meier 10-year recurrence-free disease-specific survival curves for endometrial stromal tumors with no/mild $(89 \%)$, moderate $(29 \%)$ and severe $(17 \%)$ atypia. 
A

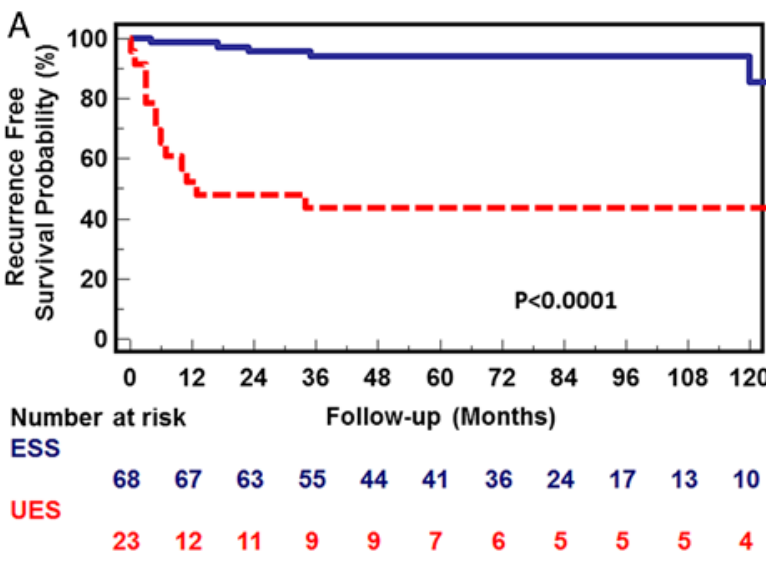

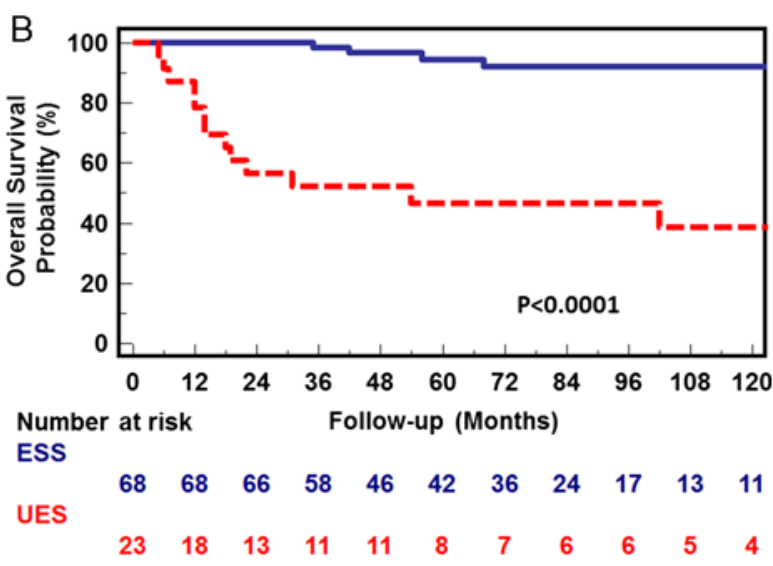

FIG. 4. The Kaplan-Meier recurrence-free (A) and overall (disease specific) survival curves (B) of cases diagnosed by the World Health Organization 2003 criteria as endometrial stromal sarcoma, low grade (ESS) or undifferentiated endometrial sarcoma (UES).

slightly: the atypia present was only none/mild as ESS, low grade, versus all other cases as UES. This resulted in $68 \mathrm{ESS}$, low grade and 23 UES cases with $93 \%$ versus $44 \%$ recurrence-free survival $(P<0.0001$, $\mathrm{HR}=10.8$, Fig. $4 \mathrm{~A})$ and $99 \%$ versus $48 \%$ diseasespecific overall mortality-free survival $(P<0.00001$, $\mathrm{HR}=45.4$, Fig. 4B). Thus, 5 ESS, low grade cases according to the first rule were classified as UES by the revised second classification rule. In support of the second rule definition, 3 of these 5 cases recurred, and 1 patient died from recurrent disease. Comparison of the prognostic outcomes of the 2 rules by multivariate analysis confirms that the second classification rule indeed is the strongest.

Comparison of the original low/high-grade ESS and the WHO 2003 diagnoses (Table 8) shows a significant difference in $2+12=14(15.4 \%)$ of the 91 cases (Cohen $\kappa=0.52$, indicative of a moderate agreement between the 2 classifications).

\section{DISCUSSION}

The objective of this study was to evaluate the criteria used by WHO 2003 to define and distinguish the various groups of malignant endometrial stromal neoplasms, tested by a large cohort of patients with a median follow-up of 5.8 years (up to $39 \mathrm{yr}$ ). We found that the WHO 2003 criteria of atypia and necrosis, when strictly defined, allow for reliable differentiation of malignant endometrial stromal tumors in ESS, low grade versus UES as prognostically strong categories. Comparison of the original low/high-grade ESS and the WHO 2003 diagnoses shows that there is only moderate agreement between the 2 classifications. Thus, the WHO 2003 is not just a name change but results in essential differences in diagnosis, prognosis, and potentially also treatment.

In the WHO 2003 description, the definition of ESS, low grade is somewhat ambiguous ("tumors without significant cellular atypia and pleomorphism"). Usually, most pathologists grade nuclear atypia as none, mild, moderate, and severe, or as low and high. In the WHO 2003 description, even though moderate atypia is not discussed, we felt it important to determine whether this middle category had important prognostic value and also determine the reproducibility of these various nuclear atypia grades and necrosis. Mild (insignificant) atypia showed minimal variation in nuclear size and shape, and nucleoli were small. Quite commonly, mild and

TABLE 6. Multivariate analysis (Cox model) to compare the prognostic value of different degrees of atypia

\begin{tabular}{lcccccccc} 
& & & & & & \multicolumn{2}{c}{$95 \%$ Confidence interval for hazard ratio } \\
\cline { 3 - 9 } Step & Atypia & Beta & $\begin{array}{c}\text { Standard } \\
\text { error }\end{array}$ & Significance & $\begin{array}{c}\text { Hazard } \\
\text { ratio }\end{array}$ & Lower & Upper \\
\hline 1 & None + mild vs. moderate + severe & 2.3 & 0.58 & $<0.00001$ & 10.0 & 3.2 & 30.9 \\
2 & None + mild + moderate vs. severe & 0.6 & 0.64 & 0.31 & 1.9 & 0.5 & 6.8 \\
\hline
\end{tabular}


TABLE 7. Comparison between 2 classification rules using nuclear atypia and prominent necrosis to prognostically classify malignant endometrial stromal tumors as ESS, low grade or UES

\begin{tabular}{|c|c|c|c|c|c|c|}
\hline \multirow[b]{2}{*}{ Classification rule } & \multirow[b]{2}{*}{ Beta } & \multirow[b]{2}{*}{$\begin{array}{l}\text { Standard } \\
\text { error }\end{array}$} & \multirow[b]{2}{*}{$\begin{array}{l}\text { Probability of } \\
\text { no difference }\end{array}$} & \multirow[b]{2}{*}{$\begin{array}{l}\text { Hazard } \\
\text { ratio }\end{array}$} & \multicolumn{2}{|c|}{$\begin{array}{l}95 \% \text { Confidence } \\
\text { interval for } \\
\text { hazard ratio }\end{array}$} \\
\hline & & & & & Lower & Upper \\
\hline $\begin{array}{l}\text { The first rule } \\
\text { ESS, low grade }=\text { None } / \text { mild or moderate nuclear atyp } \\
\text { with absence of necrosis }\end{array}$ & & & & & & \\
\hline $\begin{array}{l}\text { UES = Severe nuclear atypia combined with necrosis } \\
\text { The second rule }\end{array}$ & 0.003 & 0.66 & 0.99 & 1.0 & 0.3 & 3.7 \\
\hline $\begin{array}{l}\text { ESS = None or mild atypia combined with no necrosi } \\
\text { UES = Either necrosis present or necrosis absent but } \\
\text { with moderate or severe atypia }\end{array}$ & 2.4 & 0.73 & 0.001 & 10.8 & 2.6 & 45.5 \\
\hline
\end{tabular}

ESS indicates endometrial stromal sarcoma; UES, undifferentiated endometrial sarcoma.

no atypia were difficult to distinguish and hence were combined into a single category. Moderate atypia was defined as cases showing scattered pleomorphic nuclei, and severe atypia was classified as having many and severely pleomorphic nuclei. The absence or presence of prominent necrosis is also easily reproducible. Cases with severe atypia are easy to diagnose, and in our study half of these cases also had necrosis. Thus, both atypia and necrosis proved to be strong independent prognostic indices; however, with the incorporation of moderate atypia with severe atypia, the prognostic value increased and, when tied with prominent necrosis, became the strongest yet. Of concern is still the potential interobserver variation in the category of moderate atypia that might occur if viewed without strict rules of classification. It may be that, in the future, immunohistochemical markers e.g., proliferation biomarkers (33) or morphometric features may be of help. Until recently, morphometric assessments were tedious, but the advent and rapid spread of digital pathology will probably change this markedly.

TABLE 8. Comparison of the original low-grade or high-grade ESS classified before 2003 with the WHO 2003 diagnoses

\begin{tabular}{lrcr}
\hline & \multicolumn{2}{c}{ WHO 2003 diagnosis } & \\
\cline { 2 - 3 } & ESS & $\begin{array}{c}\text { Undifferentiated } \\
\text { endometrial sarcoma }\end{array}$ & Total \\
\hline Original diagnosis & & & \\
ESS low & 66 & 12 & 78 \\
ESS high & 2 & 11 & 13 \\
Total & 68 & 23 & 91 \\
\hline
\end{tabular}

ESS indicates endometrial stromal sarcoma; WHO, World Health Organization.
Several issues have not been addressed and are beyond the scope of this manuscript to resolve. One is the validity of considering UES as an entity distinct from ESS, low grade as opposed to being one end of the spectrum with ESS, low grade being at the other. By analogy, should Grade 3 endometrial adenocarcinoma be potentially considered as a different entity from Grade 1 endometrial carcinoma, as both have differing prognoses even though both seem to arise by the same mechanism? In contrast, evidence now points to why borderline serous tumor/Grade 1 serous carcinoma of the ovary is a completely different entity from high-grade serous carcinoma, as both seem to arise by different pathogenetic mechanisms even though they share many histomorphologic features. For example, the former is KRAS and BRAF positive, and the latter is KRAS and BRAF negative. Issue 2 is whether the WHO 2003, in eliminating the category of high-grade ESS, has simply substituted UES in its place (Old dog, New name). Finally, Issue 3 is should the category of ESS high grade be reintroduced if it is shown to differ in some fundamental way from a subset of the current UES cases? Future studies based on immunohistochemical, molecular pathology and even wholegenome sequencing may resolve these conundrums.

In conclusion, the WHO 2003 criteria for classifying endometrial stromal tumors as ESS, low grade versus UES are somewhat ambiguous; however, on the basis of our definitions for atypia grades and necrosis, our findings result in 2 prognostically strong and separate categories. The major distinguishing features are atypia that is moderate or severe on the one hand and none or mild on the other. Further, the presence of necrosis is also an important distinguishing feature. 


\section{REFERENCES}

1. Tavassoli Fattaneh A, Devilee P. World Health Organization Classification of Tumours. Pathology and Genetics of Tumours of the Breast and Female Genital Organs. Lyon: IARC Press; 2003.

2. Abeler VM, Røyne O, Thoresen S, et al. Uterine sarcomas in Norway: a histopathological and prognostic survey of a total population from 1970 to 2000 including 419 patients. Histopathol 2009;54:355-64.

3. Norris HJ, Taylor HB. Mesenchymal tumours of the uterus: a clinical and pathological study of 53 endometrial stromal tumours. Cancer 1966;19:755-66.

4. Evans HL. Endometrial stromal sarcoma and poorly differentiated endometrial sarcoma. Cancer 1982;50:2170-82.

5. Amant F, Woestenborghs $\mathrm{H}$, Vandenbroucke V, et al. Transition of endometrial stroma sarcoma into high-grade sarcoma. Gynecol Oncol 2006;103:1137-40.

6. Benito V, Lubrano A, Arencibia O, et al. Clinicopathologic analysis of uterine sarcomas from a single institution in the Canary Islands. Int J Gynaecol Obstet 2009;107:44-9.

7. Denschlag D, Masoud I, Stanimir G, et al. Prognostic factors and outcome in women with uterine sarcoma. Eur J Surg Oncol 2007;33:91-5.

8. Ghaemmaghami F, Karimi-Zarchi M, Gilani MM, et al. Uterine sarcoma: clinicopathological characteristics, treatment and outcome in Iran. Asian Pac J Cancer Prev 2008;9:421-6.

9. Koivisto-Korander R, Butzow R, Koivisto AM, et al. Clinical outcome and prognostic factors in 100 cases of uterine sarcoma: experience in Helsinki University Central Hospital 1990-2001. Gynecol Oncol 2008;111:74-81.

10. Kokawa K, Nishiyama K, Ikeuchi M, et al. Clinical outcomes of uterine sarcomas: results from 14 years worth of experience in the Kinki district in Japan (1990-2003). Int J Gynecol Cancer 2006;16:1358-63.

11. Livi L, Paiar F, Shah N, et al. Uterine sarcoma: twenty-seven years of experience. Int J Radiat Oncol Biol Phys 2003;57:1366-73.

12. Park JY, Kim DY, Suh DS, et al. Prognostic factors and treatment outcomes of patients with uterine sarcoma: analysis of 127 patients at a single institution, 1989-2007. J Cancer Res Clin Oncol 2008;134:1277-87.

13. Yoney A, Eren B, Eskici S, et al. Retrospective analysis of 105 cases with uterine sarcoma. Bull Cancer 2008;95:E10-7.

14. Barney B, Tward JD, Skidmore T, et al. Does radiotherapy or lymphadenectomy improve survival in endometrial stromal sarcoma? Int J Gynecol Cancer 2009;19:1232-8.

15. Chan JK, Kawar NM, Shin JY, et al. Endometrial stromal sarcoma: a population-based analysis. Br J Cancer 2008:99:1210-5.

16. Geller MA, Argenta P, Bradley W, et al. Treatment and recurrence patterns in endometrial stromal sarcomas and the relation to c-kit expression. Gynecol Oncol 2004;95:632-6.

17. Haberal A, Kayikçioglu F, Boran N, et al. Endometrial stromal sarcoma of the uterus: analysis of 25 patients. Eur $J$ Obstet Gynecol Reprod Biol 2003;109:209-13.
18. Leath CA. III, Huh WK, Hyde J, et al. A multi-institutional review of outcomes of endometrial stromal sarcoma. Gynecol Oncol 2007;105:630-4.

19. Shah JP, Bryant CS, Kumar S, et al. Lymphadenectomy and ovarian preservation in low grade endometrial stromal sarcoma. Obstet Gynecol 2008;112:1102-8.

20. Thomas MB, Keeney GL, Podratz KC, et al. Endometrial stromal sarcoma: treatment and patterns of recurrence. Int $J$ Gynecol Cancer 2009;19:253-6.

21. Kim WY, Lee JW, Choi CH, et al. Low-grade endometrial stromal sarcoma: a single center's experience with 22 cases. Int J Gynecol Cancer 2008;18:1084-9.

22. Li AJ, Giuntoli RL, Drake R, et al. Ovarian preservation in stage I low-grade endometrial stromal sarcomas. Obstet Gynecol 2005;106:1304-8.

23. Ma SK, Zhang HT, Wu LY, et al. Treatment and prognosis of low-grade malignant endometrial stromal sarcoma. Zhonghua Zhong Liu Za Zhi 2007;29:74-8.

24. Amant F, De Knijf A, Van Calster B, et al. Clinical study investigating the role of lymphadenectomy, surgical castration and adjuvant hormonal treatment in endometrial stromal sarcoma. Br J Cancer 2007;97:1194-9.

25. Ashraf-Ganjoei T, Behtash N, Shariat M, et al. Low grade endometrial stromal sarcoma of uterine corpus, a clinicopathological and survey study in 14 cases. World J Surg Oncol 2006;9:45-50.

26. Bartosch C, Exposito M, Lopes JM. Low-grade endometrial stromal sarcoma and undifferentiated endometrial sarcoma: a comparative analysis emphasizing the importance of distinguishing between these two groups. Int J Surg Pathol 2010;18: 286-91.

27. Landréat V, Paillocher N, Catala L, et al. Low-grade endometrial stromal sarcoma of the uterus: review of 10 cases. Anticancer Res 2008;28:2869-74.

28. Feng W, Hua K, Gudlaugsson E, et al. Prognostic indicators in WHO2003 low grade endometrial stromal sarcoma. Histopathol. (In press).

29. Kim HS, Song YS. International Federation of Gynecology and Obstetrics (FIGO) staging system revised: what should be considered critically for gynecologic cancer? J Gynecol Oncol 2009;20:135-136.

30. Prat J. FIGO staging for uterine sarcomas. Int $J$ Gynaecol Obstet 2009;104:177-8.

31. Kempson RL, Hendrickson MR. Smooth Muscle, Endometrial Stromal, and Mixed Müllerian Tumors of the Uterus. Mod Pathol 2000;13:328-42.

32. Baak JP, van Diest PJ, Voorhorst FJ, et al. Prospective multicenter validation of the independent prognostic value of the mitotic activity index in lymph node-negative breast cancer patients younger than 55 years. J Clin Oncol 2005;23: 5993-6001.

33. Popiolek D, Yee H, Levine P, et al. MIB1 as a possible predictor of recurrence in low-grade endometrial stromal sarcoma of the uterus. Gynecol Oncol 2003;90:353-7. 